\title{
Monitoring brown fat metabolic activity using microwave radiometry: antenna design and frequency selection
}

\author{
Dario B. Rodrigues \\ Thomas Jefferson University \\ Paul R. Stauffer \\ Thomas Jefferson University \\ Paolo F. Maccarini \\ Duke University
}

Follow this and additional works at: https://jdc.jefferson.edu/radoncfp

Part of the Biochemical Phenomena, Metabolism, and Nutrition Commons, and the Radiology Commons

\section{Let us know how access to this document benefits you}

\section{Recommended Citation \\ 2014 IEEE Benjamin Franklin Symposium on Microwave and Antenna Sub-systems}

This Article is brought to you for free and open access by the Jefferson Digital Commons. The Jefferson Digital Commons is a service of Thomas Jefferson University's Center for Teaching and Learning (CTL). The Commons is a showcase for Jefferson books and journals, peer-reviewed scholarly publications, unique historical collections from the University archives, and teaching tools. The Jefferson Digital Commons allows researchers and interested readers anywhere in the world to learn about and keep up to date with Jefferson scholarship. This article has been accepted for inclusion in Department of Radiation Oncology Faculty Papers by an authorized administrator of the Jefferson Digital Commons. For more information, please contact: JeffersonDigitalCommons@jefferson.edu. 


\title{
Monitoring brown fat metabolic activity using microwave radiometry: antenna design and frequency selection
}

\author{
Dario B. Rodrigues ${ }^{\mathrm{a}}$, Paul R. Stauffer ${ }^{\mathrm{a}}$, and Paolo F. Maccarini ${ }^{\mathrm{b}}$ \\ ${ }^{a}$ Radiation Oncology Department, Thomas Jefferson University, Philadelphia PA 19107, USA; \\ ${ }^{b}$ Biomedical Engineering Department, Duke University, Durham NC 27705, USA.
}

\begin{abstract}
This paper describes the use of microwave radiometry for monitoring brown fat metabolism (mBAT). The radiometric frequency band and receive antenna were optimized for coupling to 2-6 ml BAT depots in the supraclavicular region. Radiometric signal variations induced by higher metabolic activity resulting from a cold stimulus were calculated with electromagnetic and thermal simulations in a segmented computational model of the human neck including BAT. A $2.5 \mathrm{~cm}$ dia. $\log$ spiral antenna was selected as optimum using a $500 \mathrm{MHz}$ bandwidth and center frequency 2.75 GHz. Results suggest sufficient sensitivity to track subsurface temperature variations during long term mBAT monitoring for potential use in obesity and diabetes research.

Index Terms - Brown fat metabolism, log-spiral antenna, microwave radiometry, noninvasive monitoring, 3D multiphysics modeling.
\end{abstract}

\section{INTRODUCTION}

Brown adipose tissue (BAT) plays an important role in whole body metabolism and could potentially mediate weight gain and insulin sensitivity [1]. Although imaging techniques are available to detect BAT, there are currently no methods for long term monitoring of BAT metabolic activity (mBAT) to assess the effect of interventions.

Positron emission tomography-computed tomography (PET-CT) have proven most effective for locating BAT due to its high uptake of 18F-FDG [1,2]. These studies are expensive however and risks associated with radiation exposure prevent repetitive scanning. Magnetic Resonance Imaging has also been used, but is similarly expensive and poorly-suited to repetitive scan investigations [3]. Infrared thermography has demonstrated feasibility in locating BAT, but is not sufficiently sensitive to quantify small changes in thermogenesis of subsurface BAT [4].

In order to facilitate mBAT studies that might lead to new approaches in control of obesity and diabetes, there is a need for new technology that is painless, non-toxic, reliable, and inexpensive enough for repetitive use to quantify long term changes in mBAT in large patient studies. Microwave radiometry fits these requirements since it allows noninvasive collection of thermal radiation from deep tissue and can be calibrated to convert received power into a volumeaveraged measure of absolute temperature.

Our group recently developed a $2.5 \mathrm{~cm}$ dia. sensor that consists of a log-spiral antenna integrated with a miniature radiometer. The sensor was optimized for sensing changes in brain temperature in the $1.1-1.6 \mathrm{GHz}$ band [5]. The present study is focused on the redesign and optimization of the antenna and radiometric band to sense small changes in temperature at depth in the neck as a result of increased mBAT, which can be activated by a cold stimulus [2].

\section{METHODS}

\section{A. Log-spiral antenna design}

Log-spiral antennas exhibit frequency-independent properties over a wide band limited by the finite physical dimensions of the antenna [5]. The most critical parameters of the log-spiral geometry are the diameter, number of spiral turns $(N)$, dielectric properties and thickness of the substrate and matching layer coverlay, all of which are chosen to provide optimum coupling to the BAT target.

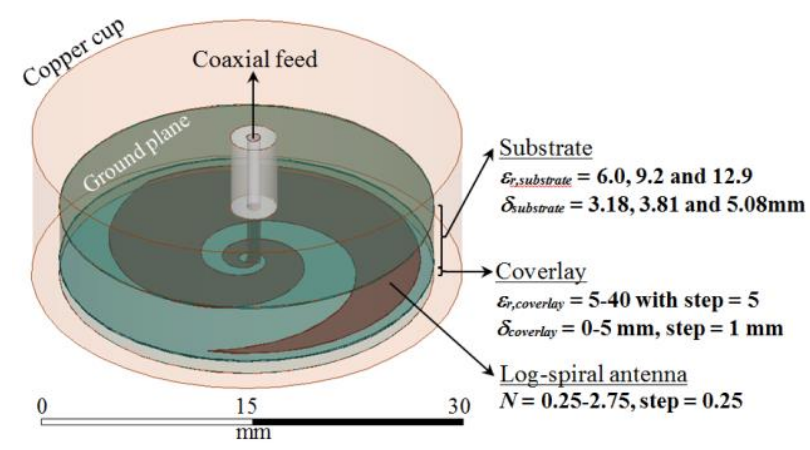

Fig. 1. Radiometric receive antenna: microstrip log-spiral patch design with tapered ends. The parameters in italic bold define the optimization strategy based on a parametric analysis, where the parameter $\delta$ represents thickness.

The microwave antenna measures an equivalent temperature $\left(T_{B}\right)$ determined from the weighted volumetric average temperature within the sense region of the antenna. The power collected by the antenna $\left(P_{a n t}\right)$ is directly proportional to $T_{B}$ and is given by $P_{a n t}=G k_{B} T_{B} \Delta f$, where $k_{B}$ is the Boltzmann constant and $G$ is the radiometer total gain. Defining $T(\boldsymbol{r})$ as the physical temperature in the human tissue located at position $r$ within a sense volume $V$, and $S_{11}$ as the power reflection coefficient due to mismatch at the antenna/load interface, the temperature measured by the radiometer is given by: 


$$
T_{B}^{j} \propto\left(1-\left|S_{11}^{j}\right|^{2}\right) \int_{V} W^{j}(\boldsymbol{r}) T(\boldsymbol{r}) d V, W^{j}(\boldsymbol{r})=\frac{S A R^{j}(\boldsymbol{r})}{\int_{V} S A R^{j}(\boldsymbol{r}) d V}
$$

where $W$ is a weighting function given by the power collection pattern which, by reciprocity [5], is the same as the specific absorption rate $\left(\mathrm{SAR}=\sigma|\boldsymbol{E}(\boldsymbol{r})|^{2} / 2 \rho\right)$, which is a function of electric conductivity $\sigma$, density $\rho$ and the electric field $\boldsymbol{E}$ inside the sense volume $V$. The superscript $j$ indicates that radiometric observations can be implemented over multiple frequency bands.

The design optimization is based on a parametric analysis (range parameters are defined in italic bold in Fig. 1) that aims to maximize the bandwidth $(\Delta f)$ of the receive antenna and efficiency $(\eta)$, which is evaluated here in terms of the ratio of power received from the brown fat (BAT) to the total power received by the antenna. Besides efficiency, the band is chosen based on the constraint $S_{11} \leq-10 \mathrm{~dB}$ in order to minimize mismatch $\left(S_{11}\right)$ between the antenna and tissue. Frequency bands allocated by the Federal Communications Commission (FCC) are specifically avoided.

\section{B. Computational model}

A 3D computational model of the human neck and chest was created in HFSS (Ansys Inc., Canonsburg, PA), from PET-CT images that were used to establish realistic volumes and locations of BAT (Fig. 2). PET-CT scans indicate volumes up to $20 \mathrm{ml}$ in lean people and approximately half that size in overweight humans. BAT regions were segmented in the supraclavicular region with a volume of 2-6 ml underlying a superficial layer of $1.5 \mathrm{~mm}$ skin and 3-10 $\mathrm{mm}$ of subcutaneous white adipose tissue (WAT). The depth of these depots ranged from 4.5-20 mm.

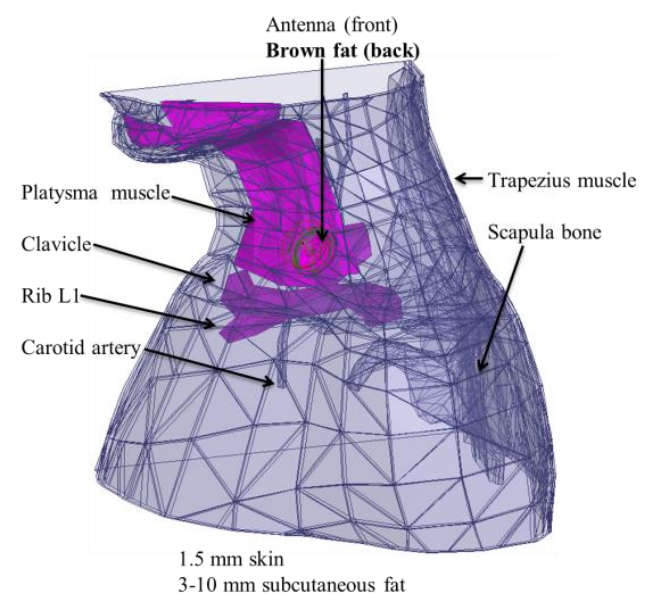

Fig. 2. Location of the antenna and 3D computational model of the neck and chest region: skin, WAT, BAT as well as relevant muscle and bone structures.

The simulation of power received by a surface antenna required thermal simulations. Heat transfer was simulated using the FEM-based software COMSOL Multiphysics
(COMSOL, Inc., Burlington MA), which solves the bioheat equation [6] that accounts for heat transfer due to conduction, blood perfusion and metabolism:

$$
\rho C_{p} \frac{\partial T}{\partial t}(\boldsymbol{r}, t)=k \nabla^{2} T(\boldsymbol{r}, t)+\omega_{b} C_{p b}\left(T_{a}-T(\boldsymbol{r}, t)\right)+Q_{m}
$$

where $C_{p}$ is specific heat capacity, $k$ is thermal conductivity, $\omega_{b}$ is volumetric blood flow rate, $T_{a}$ is arterial temperature, $Q_{m}$ is metabolic heat rate and the index $b$ stands for blood. Human tissue properties were retrieved from the literature [7]. The boundary condition at the skin surface was defined by a convection heat transfer coefficient $h_{\text {air }}=5 \mathrm{~W} / \mathrm{m}^{2} / \mathrm{K}$ and room temperature $T_{\text {air }}=25^{\circ} \mathrm{C}$ in thermoneutral conditions. The simulated power received by the antenna was characterized as a function of frequency and using different levels of mBAT under cold stimulation: $T_{a i r}=$ $15^{\circ} \mathrm{C}$.

\section{RESULTS AND DISCUSSION}

\section{A. Antenna optimization}

Simulations of $S_{11}$ and efficiency $(\eta)$ showed only minor dependence on coverlay permittivity and thickness in the analyzed ranges. Thus simulations were performed using $\varepsilon_{r, \text { coverlay }}=30$ and $\delta_{\text {substrate }}=1 \mathrm{~mm}$ due to ready availability. Simulations also showed that thicker substrates with lower dielectric permittivity provided better efficiency and larger bandwidth at the expense of larger antenna size. The same results could be obtained using thinner structures with higher dielectric permittivity providing a trade-off between efficiency and antenna size. Our strategy was to determine the optimal combination of $\varepsilon_{r, \text { substrate }}, \delta_{\text {substrate }}$ and $N$.

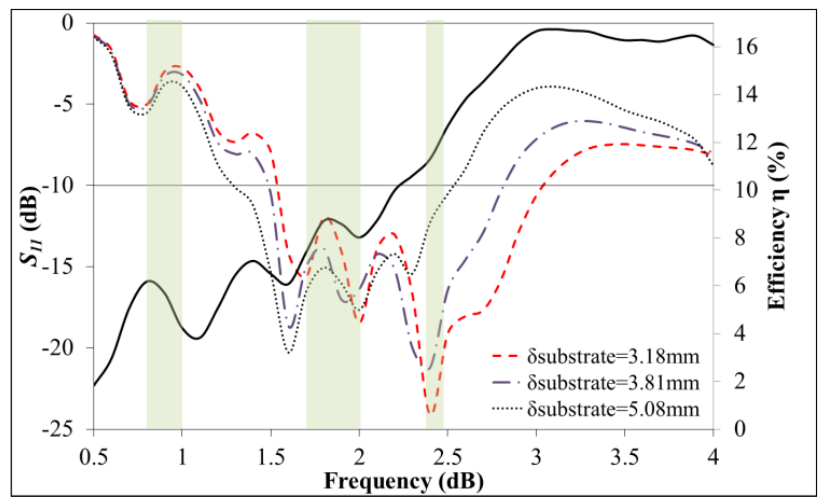

Fig. 3. Parametric analysis of substrate thickness to improve efficiency and $S_{11}$. The shaded regions represent FCC bands.

Fig. 3 shows the antenna reflection coefficient of the logspiral antenna over $0.5-4 \mathrm{GHz}$ for different substrate thicknesses. All signals present good reflection coefficients $\left(S_{11} \leq-10 \mathrm{~dB}\right)$ over a wide band as typical of log-spiral configurations. The best combination of parameters was: $\varepsilon_{r, \text { substrate }}=12.85, \delta_{\text {substrate }}=3.18 \mathrm{~mm}$ and $N=2$, which 
yielded the frequency band 2.5-3.0 GHz with an average efficiency of $15 \%$, i.e., $15 \%$ of the power is collected from the sub-surface BAT depot tissue. Fig. 4 shows the antenna radiation pattern simulated in HFSS for the optimized configuration, where we can observe that the antenna sense region penetrates beyond the BAT tissue region.

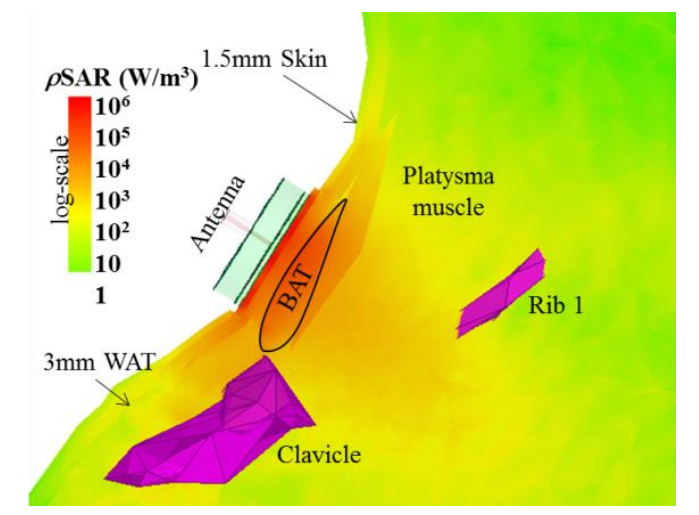

Fig. 4. Simulated radiation (receive) pattern in neck model using optimized antenna parameters and a BAT volume of $6 \mathrm{ml}$.

\section{B. Thermal modeling of BAT metabolism}

The simulated temperature and SAR were combined to compute the theoretical power received by the antenna. Fig. 5 shows the variation of received power induced by increasing mBAT up to 25 -fold with a mild cold stimulus, i.e., using $T_{\text {air }}=15^{\circ} \mathrm{C}$. It is clear that received power is dependent on the metabolic activity of different BAT depot volumes $(2-6 \mathrm{ml})$ and WAT thicknesses $(3-10 \mathrm{~mm})$. Above a 15-fold change, the received power shows less dependency on mBAT, but so far literature only describes variations in this range [1]. The correspondent average BAT temperature variation within the analyzed $\mathrm{mBAT}$ variation (5 to 25 -fold) was $0.33-1.47^{\circ} \mathrm{C}$.

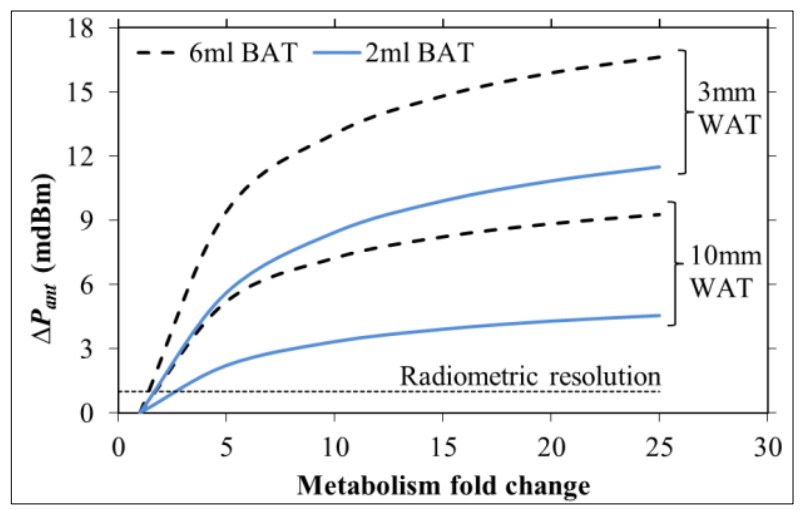

Fig. 5. Parametric simulations to assess radiometic sensitivity to mBAT variation during mild cold simulation $\left(15^{\circ} \mathrm{C}\right)$.

The minimum power variation is $2.2 \mathrm{mdBm}$ for a 5 -fold increase in mBAT ( $2 \mathrm{ml}$ BAT volume below $10 \mathrm{~mm}$ WAT thickness), which is detectable using current microwave radiometry techniques $[5,8]$. Our previous device [5] was designed for a larger and deeper target region (brain); it demonstrated $\mathrm{mdBm}$ sensitivity over an optimized frequency band of 1.1-1.6 GHz. The redesigned antenna discussed in this work is currently being implemented for operation at higher frequencies $(2.5-3.0 \mathrm{GHz})$ since BAT is more superficial than brain.

\section{CONCLUSIONS}

We presented a computational model to optimize a radiometric antenna for monitoring BAT metabolism and to estimate radiated power from typical supraclavicular BAT depots. The optimized frequency band for a $2.5 \mathrm{~cm}$ dia. thick substrate tapered log-spiral antenna was 2.5-3.0 GHz. The antenna produced good $S_{11}$ coupling and an average receive efficiency of $15 \%$ from the BAT volume. These characteristics are sufficient to detect thermal radiation from small volumes $(2-6 \mathrm{ml})$ of BAT located $4.5-20 \mathrm{~mm}$ deep and to monitor small changes $\left(<0.5^{\circ} \mathrm{C}\right)$ in BAT temperature associated with increased metabolism during mild cold exposure. The miniature sensor and a $2.75 \mathrm{GHz}$ radiometer appear suitable for non-invasive long term monitoring of BAT metabolism, enabling future experimental investigation of the role of BAT in mediating obesity and diabetes.

\section{REFERENCES}

[1] K.A. Virtanen, M.E. Lidell, J. Orava, et al., "Functional brown adipose tissue in healthy adults," N. Engl. J. Med., vol. 360, issue 15, pp. 1518-25, Apr. 2009.

[2] J. Orava, P. Nuutila, M.E. Lidell, et al., "Different Metabolic Responses of Human Brown Adipose Tissue to Activation by Cold and Insulin," Cell Metab., vol. 14, issue 2, pp. 272-9, Aug. 2011.

[3] H.H. Hu, T.G. Perkins, J.M. Chia, et al., "Characterization of human brown adipose tissue by chemical-shift water-fat MRI," Am. J. Roentgenol., vol. 200, issue 1, pp. 177-83, Jan. 2013.

[4] P. Lee, K.K.Y. Ho, J.R. Greenfield, "Hot fat in a cool man: infrared thermography and brown adipose tissue," Diabetes Obes. Metab., vol. 13, issue 1, pp. 92-3, Jan. 2011.

[5] D.B. Rodrigues, P.F. Maccarini, S. Salahi, et al., "Design and optimization of an ultra-wideband and compact microwave antenna for radiometric monitoring of brain temperature," IEEE Trans. Biomed. Eng., vol. 61, issue 7, pp. 2154-60, Jul. 2014.

[6] H.H. Pennes, "Analysis of tissue and arterial blood temperatures in the resting human forearm," J. Appl. Physiol., vol. 1, issue 2, pp. 93-122, Aug. 1948.

[7] P.A. Hasgall, E. Neufeld, M.C. Gosselin, et al. (2013, July 30). IT'IS Database for thermal and electromagnetic parameters of biological tissues (Version 2.4) [Online]. Available: http://www.itis.ethz.ch/database.

[8] K. Arunachalam, P.R. Stauffer, P.F. Maccarini, et al., "Characterization of a digital microwave radiometry system for noninvasive thermometry using a temperature-controlled homogeneous test load," Phys. Med. Biol., vol. 53, issue 14, pp. 3883-901, Jul. 2008. 